\title{
Hepatic ketone-body metabolism in developing sheep and pregnant ewes
}

\author{
BY G. CAROLE E. VARNAM, MARJORIE K. JEACOCK \\ AND D. A. L. SHEPHERD \\ Department of Physiology and Biochemistry, University of Reading, \\ Whiteknights, Reading RG6 $2 A J$
}

(Received 26 October 1977-Accepted 6 January 1978)

\begin{abstract}
I. In order to establish whether or not there is a relationship between the blood ketone-body concentrations and the potential ability of the liver to synthesize ketone bodies in sheep on varying nutritional regimens, a study has been made of the concentrations of acetoacetate and 3-hydroxybutyrate in blood and the activities of enzymes concerned with ketogenesis in liver of developing sheep from mid-way through gestation to maturity, in pregnant ewes from mid-way through pregnancy and in starved pregnant and non-pregnant ewes.

2. During development the most marked change in blood 3-hydroxybutyrate concentration occurred when the lambs were weaned. Blood acetoacetate concentrations did not change during development. When mature ewes were starved both 3-hydroxybutyrate and acetoacetate concentrations in blood were increased.

3. Changes found in the activity of 3-hydroxybutyrate dehydrogenase (EC I.I.I.30) in the liver were correlated with the changes in blood 3-hydroxybutyrate concentrations during development but no such relationship existed in pregnant or fasted ewes. No correlation was found between the ability of the liver to synthesize acetoacetate and blood ketone body concentrations in either developing or pregnant adult sheep. The rate of acetoacetate production expressed per $\mathrm{g}$ liver increased during foetal life but values observed in lambs I d after birth were similar to those found in suckling and mature sheep. During the last month of pregnancy and when non-pregnant sheep were starved the hepatic potential for ketogenesis was increased. During development the activity of acetyl-CoA acetyltransferase $(E C 2.3 .1 .9)$ was correlated with the rate of hepatic acetoacetate production.

4. These changes have been contrasted with those that occur in developing and starved adult rats.

5. It is concluded that hepatic production of ketone bodies cannot be the only factor in the regulation of blood ketone body concentrations in developing and pregnant sheep.
\end{abstract}

Comparison of results for foetal (Morriss, Boyd, Makowski, Meschia \& Battaglia, I974), I-d-old (Koundakjian \& Snoswell, I970) and adult sheep (Katz \& Bergman, I969) indicates that changes must occur in blood ketone-body concentrations during development in this species. These changes may result from changes in either the rate of production of ketone bodies or the rate of their irreversible loss. During the development of the rat Bailey \& Lockwood (1973) have found that there are marked changes in the ability of the liver to synthesize ketone bodies and this enhanced potential for ketogenesis appears to be closely related to blood ketone-body concentrations. These changes have been attributed to the varying dietary regimens of the rat at birth, during suckling and when weaned onto a high-carbohydrate diet. In the adult rat, in contrast to the newborn rat, the ketosis of starvation is not accompanied by any marked increase in the activity of the enzymes of hepatic ketogenesis (Williamson, Bates \& Krebs, I968).

In ruminants there are two major sites of ketogenesis, namely the wall of the reticulorumen and the liver. The relative amounts of ketone bodies originating from these two organs will vary with the stage of development and the nutritional status of the animal. For example, in the foetus and newborn lamb the rumen is poorly developed and the liver must be the source of endogenous ketone bodies. In well-fed non-pregnant and nonlactating mature sheep the predominant site of ketogenesis is the rumen wall, but in 
Table I. Average daily intake of metabolizable energy $(M J)$ and digestible crude protein (nitrogen $\times 6.25 ; g$ ) by the ewes

$\begin{array}{lccccc} & \begin{array}{c}\text { Pregnant ewes (d pregnant) } \\ \text { pregnant } \\ \text { ewes }\end{array} & \overbrace{64-84}^{\text {Non- }} & 85-105 & 106-126 & 127-147 \\ \text { Metabolizable energy } & 14.0 & 14.7 & 16.5 & 19.3 & 18.8 \\ \text { Digestible crude protein } & 77 & 100 & 105 & 119 & 115\end{array}$

undernourished or pregnant sheep the liver assumes greater importance in ketone-body synthesis (Katz \& Bergman, 1969).

Whether or not changes that occur in the blood ketone-body concentrations in sheep during development, pregnancy or undernutrition of mature animals are also accompanied by changes in the ketogenic enzyme profile of the liver has not been established. This paper reports the blood ketone-body concentrations, the activities of 3-hydroxybutyrate dehydrogenase (EC I. I. I .30) and acetyl-CoA acetyltransferase (EC 2.3.1.9) in the liver and the hepatic potential for ketogenesis, as indicated by the rate of acetoacetate production from acetyl-CoA, in developing sheep from mid-way through gestation to maturity, a period during which the nutrition of sheep changes markedly. The effect of pregnancy and fasting on the rate of ketogenesis from acetyl-CoA and the activity of 3-hydroxybutyrate dehydrogenase have also been studied in the livers of mature sheep.

\section{EXPERIMENTAL}

Animals and diets

Ewes used in this study were either Dorset Horn or Clun Forest. Most were tupped by Suffolk rams on known dates in order to obtain lambs of known conceptual age. The stage of gestation was confirmed and the number of foetuses carried were detected by radiological examination at the Grassland Research Institute, Hurley, Berks.

The ewes were housed indoors for at least 2 weeks prior to operation or lambing. They were given hay and concentrate daily. The concentrate fed was either a mixture of equal parts of flaked maize and barley or a commercial pelleted ration. The amounts offered to the fed non-pregnant and pregnant ewes were such that the amount of metabolizable energy (ME) and digestible crude protein (nitrogen $\times 6.25$ ) were equal to or greater than the recommended allowances (Agricultural Research Council, I965; MAFF, 1975) considering the weight of the ewe, the stage of pregnancy and the number of foetuses carried. Daily residues were weighed and the average daily intake of ME and digestible crude protein of the ewes is given in Table $\mathrm{I}$. Lactating ewes were fed hay ad lib. and I kg concentrates daily. Fasted ewes were without food for $4 \mathrm{~d}$.

Suckling lambs which were to be killed within 3 weeks of birth (i.e. animals killed between 148 and $169 \mathrm{~d}$ conceptual age) were born indoors. Prior to slaughter they suckled their mothers and had access to their mother's rations. Commercial lamb weaning pellets were available as creep feed 2 weeks after birth. Lambs slaughtered at I $89 \mathrm{~d}$ conceptual age were separated from the ewes 5 weeks after birth and fed hay and the weaning pellets ad lib. The fat lambs killed between $252-266 \mathrm{~d}$ conceptual age were at grass prior to slaughter. All animals had access to water ad lib. 


\section{Collection of liver samples and blood}

Livers from foetal lambs were obtained from animals slaughtered after delivery by Caesarian section. Livers from suckling and fat lambs were obtained from animals slaughtered after electrical stunning and samples of liver from ewes were obtained by biopsy. These techniques have been described previously (Edwards, Dhand, Jeacock \& Shepherd, 1975). The liver samples were placed immediately in ice-cold $0.15 \mathrm{M}$-potassium chloride.

Foetal umbilical arterial and venous blood samples were obtained at Caesarian section prior to severing the umbilical cord. Blood samples were obtained from the jugular vein of postnatal lambs and ewes. Blood was placed immediately into an approximately equal volume of ice-cold $0.6 \mathrm{M}$-perchloric acid.

\section{Analytical procedures}

Chemicals. Tris, ATP, NAD ${ }^{+}, \mathrm{NADH}, \mathrm{CoA}$, acetyl-phosphate (dilithium salt) and purified enzymes were obtained from Boehringher Corp. (London) Ltd, Lewes, East Sussex; 3-mercaptoethanol from Sigma (London) Chemical Co. Ltd, Kingston-uponThames, Surrey. Acetoacetate for use as a standard was prepared by the method of Hall (1962) and acetoacetyl-CoA was synthesized from coenzyme A and diketene by the method of Simon \& Shemin (1953). All other chemicals were supplied by British Drug Houses Ltd, Poole, Dorset or Fisons Ltd, Loughborough, Leicestershire and were of the highest purity available.

Blood ketone-body concentrations. Blood acetoacetate and 3-hydroxybutyrate concentrations were determined in perchloric acid extracts of blood by the enzymic method of Williamson, Mellanby \& Krebs (1962).

Hepatic acetoacetate production. Samples of liver were homogenized in a glass-tefion Potter-Elvehjem homogenizer in 20 vol. of a medium containing (/l): sucrose $0.25 \mathrm{~mol}$, 2-mercaptoethanol $\mathrm{I} \mathrm{mmol}$, Tris $10 \mathrm{mmol}$, adjusted to $\mathrm{pH} 7 \cdot 0$ with hydrochloric acid. The homogenates were sonicated for $45 \mathrm{~s}$ with intermittent bursts using a $9 \mathrm{~mm}$ probe at 6-7 kc/s, 8-9 $\mu \mathrm{m}$ amplitude (I00 W ultrasonic disintegrator; Measuring and Scientific Equipment Ltd, London) and then centrifuged for $30 \mathrm{~min}$ at $38000 \mathrm{~g}$. These procedures were carried out at $0-4^{\circ}$. Portions of the supernatant fractions were incubated with an acetyl-CoA-generating system for $10 \mathrm{~min}$ at $37^{\circ}$ in the medium described by Williamson et al. (1968) for their assay of hydroxymethylglutaryl-CoA synthase (EC 4. I.3.5) activity. The acetoacetate formed was determined enzymically (Williamson et al. 1962). Williamson et al. (1968) bave argued that this assay is a measure of the activity of hydroxymethylglutaryl-CoA synthase. Since neither the amount of the substrate used nor the immediate product formed by this enzyme are measured directly, we consider that the measurement made is best described as the rate of acetoacetate production from acetyl-CoA.

Acetyl-CoA acetyltransferase activity. The activity of this enzyme was determined in tissue supernatant fractions, prepared as for the determination of the rate of acetoacetate production, by measuring the decrease in extinction at $310 \mathrm{~nm}$ resulting from the disappearance of the acetoacetyl-CoA- $\mathrm{Mg}^{2+}$ complex in the presence of CoA (Williamson et al. 1968).

3-Hydroxybutyrate dehydrogenase activity. Tissue was homogenized in Io vol. of an ice-cold medium, adjusted to $\mathrm{pH} 7 \cdot 4$, containing (/l): succinate Io mmol, EDTA I mmol, ATP $0.6 \mathrm{mmol}$ and $\mathrm{NAD}^{+}$I mmol. The homogenates were sonicated at $0-4^{\circ}$ for $4 \mathrm{~min}$. 3-Hydroxybutyrate dehydrogenase activity was assayed by the spectrophotometric method of Lehninger, Sudduth \& Wise (I960) but 2-mercaptoethanol was used in place of cysteine. 
)

?

辛 


\section{Table 3. Umbilical arterio-venous differences for blood acetoacetate and 3-hydroxybutyrate ( $\mathrm{mmol} / \mathrm{l})$}

(Mean values with their standard errors; no. of animals in each group is given in parentheses)

\begin{tabular}{|c|c|c|c|c|c|c|c|c|}
\hline & & & & Concept & l age (d) & & & \\
\hline & & -84 & & 105 & 100 & -126 & I 27 & -147 \\
\hline & Mean & SE & Mean & SE & Mean & SE & Mean & SE \\
\hline cetoacetate & -0.011 & $0.020(3)$ & +0.014 & $0.032(3)$ & +0.018 & $0.012(5)$ & +0.001 & $0.007(8)$ \\
\hline 3-Hydroxybutyrate & -0.006 & $0.008(3)$ & +0.004 & $0.028(3)$ & -0.007 & $0.010(5)$ & +0.006 & $0.006(8)$ \\
\hline
\end{tabular}

\section{Statistical analyses}

The standard error of each mean was calculated and the statistical significance of the differences between means were assessed by Student's $t$ test. The logistic curve which indicates the relationship between the rate of acetoacetate production in ewe liver and the duration of pregnancy was derived using a maximum-likelihood programme obtained from the Statistics Department, Rothampstead Experimental Station, Harpenden, Hertfordshire by the Applied Statistics Department, University of Reading.

\section{RESULTS}

The rate of acetoacetate production from acetyl-CoA by liver homogenates, the activities of 3-hydroxybutyrate dehydrogenase and acetyl-CoA acetyltransferase in the liver and the concentrations of ketone bodies in blood of foetal, suckling and ruminating lambs aged between 64 and $266 \mathrm{~d}$ conceptual age are shown in Table 2 . There was a progressive rise in the ability of hepatic tissue to synthesize acetoacetate from acetyl-CoA in lambs from mid-way through gestation until I $d$ after birth. A similar change was observed in the pattern of activity of hepatic acetyl-CoA acetyltransferase during development. Analysis of the results from individual animals between 64 and $266 \mathrm{~d}$ conceptual age indicated that there was a significant relationship between the activity of acetyl-CoA acetyltransferase $(T)$ and the rate of acetoacetate production from acetyl-CoA $(A)$. This relationship may be described by the equation:

$$
A=0.0154 T+0.0703(r+0.612, n 37, P<0.001)
$$

However, there was no significant correlation between the ability of the liver to synthesize acetoacetate and the blood total ketone-body concentration. The activity of 3 -hydroxybutyrate dehydrogenase in the liver $(H)$ bore some relationship to the concentration of 3-hydroxybutyrate in blood $(B)$ since there was a significant regression between these two parameters described by the equation:

$$
H=0.279 B+0.165 \quad(r+0.372, n 33, P<0.05) .
$$

During development the increase in total ketone-body concentration in blood was due entirely to change in the 3-hydroxybutyrate concentration. Hence the percentage of ketone bodies present as acetoacetate declined from a mean value of 43 in foetal lambs to a mean of 20 in suckling lambs and 6 in mature animals.

During the last half of gestation the concentrations of acetoacetate and 3-hydroxybutyrate in foetal umbilical venous blood were not significantly different from those observed in the umbilical artery (Table 3 ). 


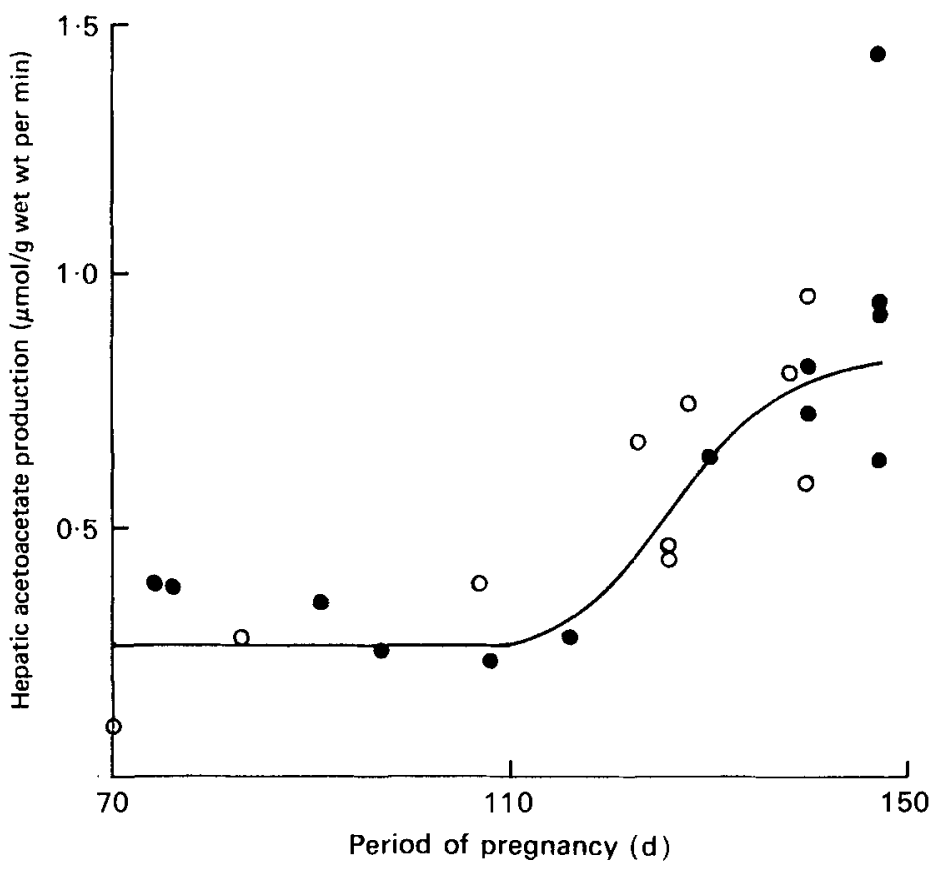

Fig. I. The relationship between the rate of acetoacetate production $(\mu \mathrm{mol} / \mathrm{g}$ wet weight per min) from acetyl-CoA by liver homogenates obtained from ewes during the second half of pregnancy and the period of pregnancy (d). $O$, Ewes carrying one foetus; $\bullet$, ewes carrying two foetuses. The equation describing the fitted curve shown is:

$A=0.266+0.614 \exp (-e(-0.125(D-124.3)))$, where $\mathrm{A}$ is the rate of acetoacetate production ( $\mu \mathrm{mol} / \mathrm{g}$ wet weight per $\mathrm{min}$ ) and $\mathrm{D}$ is the period of pregnancy (d).

Table 4. The rate of hepatic acetoacetate production, the activities of hepatic 3-hydroxybutyrate dehydrogenase (EC I I I I .30) and hepatic acetyl-CoA acetyltransferase (EC 2 . 3 . . .9) and the total ketone-body concentration in jugular blood of fed and $4 \mathrm{~d}$-fasted non-pregnant and twin-pregnant ewes

(Mean values with their standard errors; no. of animals in each group is given in parentheses)

\begin{tabular}{|c|c|c|c|c|c|c|c|c|}
\hline & \multicolumn{4}{|c|}{ Non-pregnant } & \multicolumn{4}{|c|}{ Twin-pregnant $\dagger$} \\
\hline & \multicolumn{2}{|r|}{ Fed } & \multicolumn{2}{|c|}{ Fasted } & \multicolumn{2}{|c|}{ Fed } & \multicolumn{2}{|c|}{ Fasted } \\
\hline & Mean & SE & Mean & SE & Mean & SE & Mean & SE \\
\hline $\begin{array}{l}\text { Acetoacetate production } \\
(\mu \mathrm{mol} / \mathrm{g} \text { liver per } \mathrm{min})\end{array}$ & 0.39 & $0.06(10)$ & $1 \cdot 06^{* *}$ & $0.16(6)$ & $0.99^{* *}$ & $0.17(4)$ & $\mathrm{I} \cdot 04^{* * *}$ & $0.08(4)$ \\
\hline $\begin{array}{l}\text { 3-Hydroxybutyrate de- } \\
\text { hydrogenase (units } \ddagger / g \\
\text { liver) }\end{array}$ & 0.404 & $0.032(10)$ & 0.319 & $0.042(10)$ & 0.335 & $0.008(3)$ & 0.371 & $0.052(4)$ \\
\hline $\begin{array}{l}\text { Total ketone bodies } \\
\text { (mmol/1 blood) }\end{array}$ & 0.52 & $0.15(20)$ & $I \cdot 9 I^{* *}$ & $0 \cdot 36(4)$ & 0.75 & $0.09(4)$ & $6 \cdot 12^{*}$ & $2 \cdot 28(4)$ \\
\hline
\end{tabular}


Fig. I shows that the rate of acetoacetate production from acetyl-CoA by livers obtained from pregnant ewes rose markedly during the last month of pregnancy. During the 3 rd and $4^{\text {th }}$ month of pregnancy the rate of acetoacetate production from acetyl-CoA by maternal liver was similar to that found in the fat lambs. There was no difference in the rate of acetoacetate production by liver homogenates prepared from ewes bearing one lamb compared with those prepared from ewes bearing twins.

When pregnant ewes bearing twins were fasted for $4 \mathrm{~d}$ near term there was no change in the ability of liver homogenates to synthesize acetoacetate, whereas when non-pregnant ewes were fasted for a similar period the potential ketogenic rate was increased from values similar to those found in fat lambs to values similar to those found in twin-pregnant ewes near term (Table 4 ). The activity of 3-hydroxybutyrate dehydrogenase in the livers of adult ewes was similar to that found in the fat lambs and was not affected by either pregnancy or fasting. Compared with fed non-pregnant ewes the blood total ketone-body concentration was not significantly different in twin-pregnant fed ewes, whereas fasting for $4 \mathrm{~d}$ resulted in significant increases in blood total ketone-body concentration in both non-pregnant and twin-pregnant sheep. During fasting the percentage of total ketone bodies present as acetoacetate increased from 5 to 20 in both non-pregnant and twin-pregnant animals.

\section{DISCUSSION}

The values for blood ketone-body concentrations found in this study are similar to those reported by various groups of workers who have studied foetal (Morriss et al. 1974), I-d-old (Koundakjian \& Snoswell, 1970) and adult sheep (Katz \& Bergman, 1969). The ketone bodies found in foetal blood are likely to be synthesized in foetal liver since the rumen is poorly developed in the foetus and a comparison of ketone-body concentrations in umbilical venous and arterial blood indicates there is no significant release of ketone bodies from the placenta into the foetal circulation. The absence of significant umbilical venous-arterial differences for acetoacetate and 3-hydroxybutyrate in lambs during the second half of gestation indicates that ketone bodies of either placental or maternal origin do not contribute substantially to the nutrition of the foetal lamb. This finding is in accord with that reported by Morriss et al. (1974) for foetuses aged more than II3 d. Katz \& Bergman (1969) have pointed out that when the liver is the major site of ketone-body production in adult sheep the proportion of total blood ketone bodies present as acetoacetate is at least $20 \%$. Our results for fasted adult sheep are in accord with this and the fact that in foetal lambs acetoacetate accounts for more than $40 \%$ of the total ketone body concentration is further indication that the liver is the only site of ketogenesis before birth.

Whilst there is a twofold difference in blood total ketone-body concentrations between mature foetal and I-d-old lambs it is only after weaning on to a diet including hay that the high blood ketone-body concentrations characteristic of fed adult ruminants are achieved. These changes during development in sheep may be contrasted with those which occur in the developing rat (Foster \& Bailey, 1976) where the increase in blood total ketone-body concentration after birth is four times greater than the post-partum rise in lambs. In suckling rats blood ketone-body concentration is greater than $0.7 \mathrm{mmol} / \mathrm{l}$ but after weaning on to a high-carbohydrate diet the concentration declines whereas in the sheep it is at weaning that the blood 3-hydroxybutyrate concentration rises. It has been argued that these changes in blood ketone-body concentrations in the rat result from changes in the nutritional regimen. In particular the ketosis found in suckling rats has been attributed to the high fat content of the diet (Lockwood \& Bailey, 197I). Whilst the initial rise after birth may be due to transient starvation in both species, the differences in blood ketone-body concentrations in the rat and sheep during the suckling phase may 
be linked with the fact that the fat content of rat milk is $148 \mathrm{~g} / \mathrm{kg}$ whereas that of sheep milk is only $53 \mathrm{~g} / \mathrm{kg}$ (Schmidt, 197I). The increase in the blood 3-hydroxybutyrate concentration in sheep after weaning may be related to the development of the rumen which results in the carbohydrate content of the ration being fermented so that fatty acids are absorbed rather than sugars as in the weaned rat.

In the developing sheep from mid-way through gestation until I $d$ after birth there was a progressive rise in the ability of the liver to synthesize acetoacetate from acetyl-CoA. During postnatal development in fed sheep there was no further significant change in the ketogenic potential per unit weight of liver. The rate of acetoacetate synthesis from acetylCoA by liver homogenates obtained from suckling lambs is similar to the rate of ketonebody production from butyrate by liver slices obtained from lambs aged between birth and $60 \mathrm{~d}$ reported by Hird \& Weidemann (1964) when allowance is made for the water content of liver. It is therefore apparent that the rate-limiting reaction in the conversion of butyrate to ketone bodies in lambs of this age is neither the activation of butyrate nor the conversion of butyryl-CoA to acetyl-CoA.

It is apparent that during development there is no relationship between the ability of the liver to synthesize acetoacetate and blood total ketone-body concentration. This finding in the sheep is different from the changes reported by Bailey \& Lockwood (1973) in developing rats. The increase in acetoacetate production from acetyl-CoA in rat liver at birth and the decrease at weaning has been attributed to changes in the mitochondrial activity of hydroxymethylglutaryl-CoA synthase, the rate-limiting enzyme for ketone-body synthesis. It has been suggested that the activity of this enzyme in the rat is related to the plasma lipid concentration and the fat content of the rat's diet (Lockwood \& Bailey, I97I; Williamson et al. 1968). Since the fat content of sheep milk is only $36 \%$ of that in rat milk it is not surprising that there are no significant changes in ketogenic potential of sheep liver during suckling if the maintenance of high hydroxymethylglutaryl-CoA synthase activity depends on a high dietary fat intake in mammals.

In adult ewes the capacity of the liver to synthesize acetoacetate may be influenced by either pregnancy or fasting. Despite the fact that the pregnant ewes were well-fed there was an increased hepatic ketogenic potential during the last month of pregnancy. Since there was a concomitant change in the ability of foetal liver to synthesize ketone bodies it is possible that the change is induced by a common factor in both mother and foetus. As in the foetus, this enhanced potential for ketogenesis in pregnant ewes did not give rise to raised ketone-body concentrations in blood. Fasting of twin-bearing ewes for $4 \mathrm{~d}$ in the last week of pregnancy did not further increase the ability of the liver to synthesize acetoacetate, although a similar period of fasting in non-pregnant animals resulted in a $270 \%$ increase in hepatic ketogenic potential to values similar to those found in late pregnancy. This change observed when non-pregnant ewes were fasted was considerably greater than the $68 \%$ increase in hepatic hydroxymethylglutaryl-CoA synthase activity found in male rats starved for $48 \mathrm{~h}$ (Williamson et al. I968). The maximum hepatic ketonebody production rate expected in vivo in fed and fasted twin-bearing ewes can be calculated from the rates of acetoacetate production observed in liver homogenates. Assuming a liver weight of $800 \mathrm{~g}$, these values are $47.5 \mathrm{mmol} \mathrm{ketone}$ bodies/h and $49.9 \mathrm{mmol} \mathrm{ketone}$ bodies $/ \mathrm{h}$ respectively. The value for the fasted animal is similar to the net total ketone-body production of $43.7 \mathrm{mmol} / \mathrm{h}$ observed in conscious, cannulated, fasted twin-pregnant ewes by Katz \& Bergman (1969), and it is therefore apparent that the potential is almost fully utilized in these animals. In fed twin-pregnant ewes the potential is four times greater than the production rate observed in vivo.

Acetyl-CoA acetyltransferase catalyses the interconversion of acetyl-CoA and acetoacetyl-CoA and this interconversion is involved in the catabolism of fatty acids and the 
biosynthesis of steroids as well as ketogenesis. However, the significant correlation between the activity of this enzyme and the synthesis of acetoacetate from acetyl-CoA implies that a major role for this enzyme in the liver of developing sheep is in ketone-body synthesis.

The activity of 3-hydroxybutyrate dehydrogenase in the liver of the sheep during development does not appear to be related to the ability of the liver to synthesize acetoacetate. It is notable that the higher activities of this enzyme are found in fed ruminating animals when the rumen wall is the major source of circulating ketone bodies. Fasting of adult sheep whether pregnant or not, although resulting in raised blood 3 -hydroxybutyrate dehydrogenase concentrations, did not alter hepatic 3-hydroxybutyrate dehydrogenase activity, as was also reported by Watson \& Lindsay (1972). Thus it is probable that the change in enzyme activity during development is related to some factor associated with the change in the nutrition of the animal at the onset of rumination other than the blood ketone-body concentrations.

The concentration of any metabolite in blood is a consequence of the relative rates of production and utilization. The fact that there is no correlation between the potential for hepatic ketone-body production and blood ketone-body concentrations in sheep suggests that the liver is of little importance in the regulation of blood ketone-body concentration in well-fed sheep during development and pregnancy. Whether or not the activities of ketone-body-utilizating enzymes in extrahepatic tissues may be more closely related to blood ketone-body concentration in sheep is a matter for further investigation.

This investigation was supported in part by a grant from the Agricultural Research Council. G.C.E.V. was a recipient of a postgraduate studentship from the Ministry of Agriculture, Fisheries and Food. The authors thank Mr I. A. N. Wilson of the Grassland Research Institute, Hurley, Berks. who carried out the radiological examination of the pregnant sheep.

\section{REFERENCES}

Agricultural Research Council (1965). The Nutrient Requirements of Farm Livestock, No. 2. Ruminants. London: HM Stationery Office.

Bailey, E. \& Lockwood, E. A. (1973). Enzyme 15, 239.

Edwards, E. M., Dhand, U. K., Jeacock, M. K. \& Shepherd, D. A. L. (1975). Biochim. biophys. Acta 399, 217.

Foster, P. C. \& Bailey, E. (1976). Enzyme 21, 397.

Hall, L. M. (1962). Analyt. Biochem. 3, 75.

Hird, F. J. R. \& Weidemann, M. J. (I964). Biochem. J. 93, 423.

Katz, M. L. \& Bergman, E. N. (1969). Am J. Physiol. 216, 953.

Koundakjian, P. P. \& Snoswell, A. M. (1970). Biochem. J. Ir9, 49.

Lehninger, A. L., Sudduth, H. C. \& Wise, J. B. (1960). J. biol. Chem. 235, 2450.

Lockwood, E. A. \& Bailey, E. (197I). Biochem. J. 124, 249.

MAFF (1975). Tech. Bull. Minist. Agric. Fish. Fd. no. 33.

Morriss, F. H., Boyd, R. D. H., Makowski, E. L., Meschia, G. \& Battaglia, F. C. (I974). Proc. Soc. exp. Biol. Med. $\mathbf{1 4 5}, 879$.

Schmidt, G. H. (1971). Biology of Lactation. San Francisco: W. H. Freeman.

Simon, E. J. \& Shemin, D. (1953). J. Am. Chem. Soc. 75, 2520.

Watson, H. R. \& Lindsay, D. B. (1972). Biochem. J. 128, 53.

Williamson, D. H., Bates, M. W. \& Krebs, H. A. (I968). Biochem. J. 108, 353.

Williamson, D. H., Mellanby, J. \& Krebs, H. A. (1962). Biochem. J. 82, 90. 